Sémiologie, innovation et entreprise - Colloque de l'Anvie 9-10 décembre 1998

\title{
Blandine Arondel
}

\section{(2) OpenEdition}

12 Journals

Édition électronique

URL : http://journals.openedition.org/communicationorganisation/2239

DOI : 10.4000/communicationorganisation.2239

ISSN : $1775-3546$

Éditeur

Presses universitaires de Bordeaux

Édition imprimée

Date de publication : 1 mai 1999

ISSN : 1168-5549

Référence électronique

Blandine Arondel, « Sémiologie, innovation et entreprise - Colloque de l'Anvie 9-10 décembre 1998 », Communication et organisation [En ligne], 15 | 1999, mis en ligne le 26 mars 2012, consulté le 19 avril 2019. URL : http://journals.openedition.org/communicationorganisation/2239; DOI : 10.4000/ communicationorganisation.2239

Ce document a été généré automatiquement le 19 avril 2019

(c) Presses universitaires de Bordeaux 


\title{
Sémiologie, innovation et entreprise - Colloque de l'Anvie 9-10 décembre 1998
}

\author{
Blandine Arondel
}

1 «Sémiologie, Innovation et Entreprise» tel était le thème du colloque des 9 et 10 décembre qui faisait suite à un premier colloque organisé par l'ANVIE ${ }^{1}$ en février 1996, intitulé "Sémiologie et Entreprise», ouvrant dès lors le débat sur la présence et les applications de cette discipline universitaire au champ pragmatico-commercial de l'entreprise.

2 Étaient présentes près de 250 personnes. Les intervenants, au nombre de 50, étaient des professionnels (consultants, responsables d'entreprises) et d'universitaires.

3 Les organisations représentées étaient les suivantes: Sorgem, Arkema, Sygil, Humalogie, Média System, EM, Business Marques (cabinets d'études et de marketing), Intégral concept (groupe de design), Critt crisalide (Centre Régional d'Innovation et de Transfert de Technologie), CRAM Ile de France, EDF, RATR SNCF, IBM, Carrefour, Yves SaintLaurent.

4 La grande surprise de ce colloque fut de voir se côtoyer et se confronter non seulement professionnels et universitaires mais aussi une grande variété de secteurs d'activités. Les fexposés étaient en effet aussi divers que : l'introduction sur le marché d'un distributeur de pommes dans les collèges, les plans de communication des homologues européens d'EDF, l'accueil des visiteurs dans l'entreprise en fonctionnement, la supervision sociale, la mise en place de la publicité dans le métro parisien, l'intégration des nouvelles technologies dans le secteur agro-alimentaire, la campagne publicitaire d'un «alicament», le packaging des produits Carrefour... mais aussi des variations moins pratico-pragmatiques (conçues par des universitaires) sur des thèmes tels que: la publicité des montres : modernité et antiquité d'un mythe ; l'épopée, modèle sémiotique de l'innovation; mythologies, informatique et métaphore; les enjeux de l'innovation au miroir du discours sur l'emploi; l'Euro: stratégies et conditions d'une innovation annoncée. 
5 Autant de sujets que de conceptions et d'approches différentes du concept de l'innovation.

\section{Pour une meilleure acuité}

6 La sémiologie, ou la sémiotique, selon les diverses acceptions souvent polémiques que chaque terminologie recouvre, est une discipline très récente, inspirée par la linguistique (Saussure) et formalisée initialement par le philosophe américain Ch. Peirce. Ce n'est seulement qu'à partir des années soixante qu'elle tend à s'institutionnaliser, dans un cadre strictement universitaire. Science de l'interaction du sens des signes, la sémiologie tend depuis une trentaine d'années à s'infiltrer dans le champ des organisations. En tant qu'élément d'analyse prospective, elle s'inscrit comme support de la conception d'un travail le plus souvent rattaché à la communication, au marketing, à la publicité ou au design. La sémiologie permet alors une grille d'analyse, susceptible d'encoder une signification concernant par exemple l'émission d'un nouveau produit au sein d'un contexte concurrentiel. En tant qu'élément d'analyse rétrospective, la sémiologie compare l'émission et la réception du produit ou du service dont il est question, ajuste l'analyse de l'émission ou de la réception en pondérant et hiérarchisant les éléments qui font sens.

7 Ainsi, par cette entremise, la sémiologie tente d'apporter une meilleure acuité à un ensemble systémique.

8 L'originalité (et l'intérêt) de la sémiologie est de prendre en compte la totalité des discours. Un discours pouvant être induit par une image, un objet, une architecture, une enquête d'usagers, une intention marketing, un refus d'adhésion.

\section{Saisir l'innovation...}

9 Le thème lancé étant l'innovation, il s'est agi durant ce colloque d'entrevoir la contribution de la sémiologie quand l'entreprise veut ou croit innover, et par ricochet, le rôle que peut jouer l'entreprise dans le processus d'innovation sémiotique.

10 Les variations autour de l'acception de ce terme « innovation » ont été riches et variées. L'apport des universitaires fut précieux, quand les contraintes de la loi du marché et de la concurrence incitent fortement à confondre " changement » et « innovation ", " nouveau produit » et « produit innovant ».

11 Aujourd'hui, on ne parle plus du progrès, mais de l'innovation. La résonance terminologique se déplace et il y aurait un changement de valeur induite pour tout ce qui concerne «le nouveau ». Cependant la force illocutoire de l'innovation se saisit avec des pincettes : l'innovation ne serait pas à assimiler à un simple «changement " (de nom d'entreprise, de monnaie, de logo ou de packaging). Elle nécessite une véritable rupture.

12 Four M. Costantini (Université Paris 8), "innover, c'est transgresser une synchronie donnée pour créer un événement nouveau ; avec succès : la nouveauté est acceptée; sans succès ": il n'y a pas de nouveauté. En d'autres termes, s'il n'y a pas de rupture avec l'existant, l'innovation est un leurre. Innover, c'est introduire dans une chose établie quelque chose de nouveau, d'encore inconnu (Dictionnaire Robert). Selon R. Ringoot (Université du Mirail Toulouse et de IUT de Lannion), l'innovation, à opposer à la création, présuppose une transformation 
de quelque chose de déjà existant, ou de non isolé. L'innovation "rénoverait » en induisant un «nouveau » dans la perception. Pour J. Fontanille (Université de

Limoges, Institut Universitaire de France), l'innovation serait avant tout une instance d'énonciation, au sein de laquelle ce serait l'usager l'énonciateur, le sujet de la structure actantielle: "l'innovation en entreprise ne peut exister en tant que telle que par rapport à des usagers, des consommateurs ».

Difficile, en effet, de saisir le schème de l'innovation, dans le fracas d'emphase discursive dont nous sommes quotidiennement inondés, au sein d'une surabondance de "nouveau " qui, en définitive, n'induit aucun changement véritable, mais seulement une certaine redondance ; quand la technologie n'apporte pas l'innovation souhaitée en amont, mais seulement une itération caduque car en décalage avec la régression sociale...

Les avis ont convergé, d'une part, pour aborder l'innovation davantage comme un « état » - état 2 (état 1 - transformation - état 2) - qu'un « faire » qui serait la transformation elle-même, ce qui place l'usager, le consommateur (potentiel) en tant que sujet d'un parcours actantiel, comme le soulignait dès le début du colloque J. Fontanille.

D'autre part, l'innovation se situerait toujours dans une visée prospective, qu'il s'agisse de l'entreprise ou du consommateur, impliquant par là même une prise de risque. "Ce risque a une face positive, interprète J. Fontanille, dans la mesure où le spectacle offert par une entreprise qui prend le risque d'innover donne matière à une refondation du contrat de confiance entre la marque et le consommateur, entre l'entreprise et l'usager. Mais il a aussi une face négative, car «l'identité » de la marque ou de l'entreprise est déstabilisée. La sémiotique permet de contrôler la cohérence: cohérence horizontale, entre l'avant et l'après de l'innovation, et cohérence verticale, entre toutes les dimensions (techniques, symboliques, visuelles, etc.) d'une même innovation ».

\section{L'innovation sémiologique, au cœur du processus innovant de l'entreprise}

Dans l'univers de la consommation, les clients sont actuellement contraints à ce que les communicateurs nomment le «double bind», exprimant le paradoxe, la double contrainte, entre le besoin de sécurité et le besoin de changement, dont les discours sociaux se font l'écho : «Faites ce qu'on vous dit, c'est la preuve de votre initiative ! »

La difficulté de l'innovation se situe précisément ici, le discours d'une marque ne pouvant être réduit à une construction de sens impliquant un schéma de persuasion univoque. L'innovation peut difficilement exister ex-nihilo car les consommateurs ont besoin de retrouver la trace d'un schème existant et en même temps le «nouveau » est, pour le consommateur, rédhibitoire.

En ce qui concerne la consommation de produits alimentaires, J.-J. Boutaud (Université de Bourgogne), affirme que «l'apparition de nouveaux produits alimentaires ne se limite pas à la transformation d'une forme existante mais crée un nouveau mode de relation avec le consommateur, sur un territoire d'utilisation non exploré jusqu'alors». La démonstration s'appuie sur le lancement d'« Actimel», nouveau produit de la famille des alicaments dont la campagne publicitaire a axé l'isotopie sur la santé, condensée dans " un même faire » (le geste médical et le geste alimentaire). «Actimel. Le nouveau geste santé du matin ", signature du produit, permet ainsi toute une série de déclinaisons permettant une version télévisuelle («Avez-vous pris votre Actimel?»; «Donnez-lui Actimel»), rappelant ainsi cette isotopie, modèle conçu sur le même mode des publicités 
homéopathiques, avec le rappel du geste de la prise médicamenteuse le matin. Ce travail de sémiologie a donc permis de saisir l'innovation d'Actimel dans sa conception publimarketing, innovation qui se rapporte en fait à la relation d'usage du consommateur, l'annonceur étant celui qui va tracer son trajet énonciatif.

A. Alessandrini, du Critt Crisalide, a analysé l'introduction des nouvelles technologies du secteur agro-alimentaire, comme les produits non pesticides. Un thème particulièrement d'actualité, où la résistance s'avère tenace, notamment du côté des producteurs et des usagers l'on préférera majoritairement des tomates que l'on sait provenir d'un champ même si elles sont traitées plutôt que celle poussées en serre avec des produits biologiques non polluants. A fortiori, ces actants ne sont pas les sujets agents auprès desquels le changement passe pour une innovation. La sémiologie innove en schématisant ces résistances, croisées avec l'intention émettrice. Le privilège est qu'elle ne censure aucun discours: les discours, même elliptiques, de tous les protagonistes sont pris en compte. La visée: atteindre une meilleure maîtrise de l'image revendiquée, émise et perçue des protagonistes, de la technologie innovante et enfin des produits concernés par cette technologie.

C. Bitoun et $\mathrm{F}$. Maier, ont expérimenté une méthode nommée "stratégimage ", au sein du groupe «Économie Industrielle» rattaché à EDF. Cette méthode permet, depuis 1993, d'enrichir l'offre de service du groupe EI, en intégrant un diagnostic sémiologique des potentialités stratégiques d'entreprises situées sur leur marché concurrentiel. Cette méthode s'appuie sur le postulat que la communication, notamment concernant les rapports d'activités, est un révélateur de l'état de santé de l'entreprise. Elle prône qu'en examinant, sur un plan sémiologique, les fondements et l'évolution d'un dispositif de communication, on peut effectuer un diagnostic de cette entreprise: culture, organisation, aspirations, capacités ou dysfonctionnements, niveau de compétitivité, etc. Cette méthode a d'ailleurs été appliquée avec succès, notamment sur les groupes tels que Westinghouse, General Electric, Siemens, GEC-Alstom, Alcatel, Framatome, en donnant même des scénarios d'anticipation sur le développement de ces entreprises.

L'exemple illustratif de la méthode se base sur l'analyse des rapports d'activités de cinq homologues européens d'EDF : National Power, RWE, ENDESA, ENEL, Tractebel. À partir du traitement singulier (relatif à la structure interne d'un rapport par exemple) ou en série des communications de chaque groupe, la méthode "stratégimage " a pu faire apparaitre les codes formels et expressifs, les plus spécifiques, les plus récurrents, pour l'ensemble de la communication de chaque groupe. En croisant l'organisation visuelle (codes couleurs, cadrages, graphismes, traitement des photos) et discursive (structure sémiolinguistique des textes...), la méthode a pu permettre de cerner les comportements stratégiques ainsi que les potentialités d'adaptation de ces entreprises à leur environnement, en identifiant les valeurs clefs de la culture d'entreprise de chaque « acteur électricien ».

En général, ce genre de travaux vient enrichir et corroborer l'analyse effectuée par l'équipe de communication, en la prolongeant par une mise en perspective sur le long terme. En terme d'innovation, "stratégimage prétend être innovante, dans la mesure où elle alimente le processus de décision propre à EDF, dans le cas de l'étude récente de ses homologues européens. [...] C'est une méthode novatrice, à la croisée de deux champ (sémiologie et entreprise) qui a priori n'avaient rien de commun ». mise en marché des produits de la marque Carrefour, tant alimentaires que non- 
alimentaires. L'enjeu pour le commanditaire consistait à produire le langage le plus approprié pour signifier la nouveauté d'une offre exprimée par une marque d'enseigne, au sein d'un univers concurrentiel déjà occupé et structuré par les discours préexistants des autres marques. Pour donner un exemple concret d'une application sémiotique au sein de l'univers de la consommation, voici le cheminement de sa démarche. Pour commencer, il se basa de manière précise sur les packagings de plus de 300 catégories de produits, en identifia le système de valeurs et la façon de signifier l'innovation, et en dégagea quelques règles pratiques, "briefs efficaces et procédures fiables" pour les agences de création type design commercial. Dans le jargon du sémioticien, le système de valeurs communes aux "marques-enseignes » est constitué par la superposition d'un programme d'usage et d'un programme de vie qui s'inscrivent dans l'axiologie des valeurs de consommation. "La vie du consommateur Carrefour est positive parce que sa consommation est positive (programme de vie). Celle-ci tire sa positivité de l'acquisition d'un produit aux qualités intrinsèques explicites et sans équivalent pour le prix proposé (programme d'usage) ». L'objectif fut d'introduire cette isotopie générique dans l'expression du récit packaging des produits de l'enseigne. Pour cela, F. Bobrie a procédé à l'identification de la typicité et de la différence de chaque catégorie de produit comme de chaque marque du produit concerné, afin d'en dégager les valeurs allant fonder l'innovation conceptuelle des packagings Carrefour. Ainsi, à partir de carrés sémiotiques typiques des discours de consommation, un travail comparatif a eu lieu en collaboration avec le chef du produit concerné et la responsable du développement packaging, afin de définir la direction pour laquelle les designers de Carrefour allaient devoir opter (mise en valeur soit de la marque et des dénominations, soit des textes d'allégation, soit des illustrations, ou encore de la signalétique...) pour que les consommateurs se sentent au plus près du produit (au niveau de sa valeur d'usage comme au niveau de sa valeur de représentation). Ce travail aura permis, selon F. Bobrie, "outre de maitriser, qualitativement, l'évolution et le changement des valeurs à exprimer par les récits packaging, mais au delà : permettre une meilleure productivité du travail de création graphique et des gains substantiels dans l'efficacité de la sélection des maquettes et visuels définitifs ».

B. Heilbrunn, consultant chez E.M. Lyon, a communiqué sur l'apport de la sémiotique tant à la réflexion qu'aux pratiques liées à l'innovation dans le domaine des marques. Selon lui, l'approche marketing considère souvent que la gestion de l'identité d'une marque consiste à développer puis à répéter, dans le temps et dans l'espace, un ensemble de signaux de reconnaissance de la marque. Récusant cet aspect statique du développement stratégique d'une marque, dans la mesure où « rester le même ce n'est pas garder son identité, c'est au contraire la dissoudre", une rupture doit émerger, afin "de susciter une force d'innovation. Même si l'identité implique la permanence et la continuité dans le temps (processus dialectique entre l'idem et l'ipse), elle ne présuppose aucune fixité, aucune rigidité. Soi-même comme un autre» (Paul Ricœur), cela implique une force d'inertie comme une force d'innovation. Les façons d'innover pour une marque sont alors variées. Cela peut être, par exemple, proposer une nouvelle façon de regarder un objet, matériel ou immatériel (exemple de Swatch qui a défini ses produits comme « des accessoires de mode qui donnent l'heure » en cassant les codes publicitaires en vigueur ; la marque OMO qui a pendant très longtemps instauré des codes de publicité lessivière puis les a cassés, lors de sa campagne présentant des singes etc.). Mais le succès d'une marque dépend avant tout de la solidarité des plans du langage de la marque, c'est-à-dire la forme de son expression (face perceptible: esthétique, utilisation des médias...) et la forme de son contenu (face intelligible: cadre conceptuel de référence, approche philosophique...). 
L'articulation de ces deux niveaux permettrait de comprendre comment une marque peut évoluer tout en restant elle-même. En étudiant les marques Montblanc et Bic, B. Heilbrunn a pu vérifier le bon fonctionnement de l'invariant, Montblanc ayant su transférer son savoir faire de l'univers de l'écriture vers celui de la maroquinerie, en maintenant une relation très forte entre les produits (plan de l'expression) de la marque et les valeurs qui fondent la vision du monde de la marque (plan du contenu), contrairement à Bic qui, en lançant le parfum Bic en 1988 a rompu le contrat fiduciaire entre la marque et les acheteurs potentiels en ne gardant qu'un seul plan du langage (celui du contenu) : seul le bas prix demeurait, constituant en outre une seule valeur de la marque.

L'ensemble de ces cas de figure renverrait par conséquent à une innovation partagée entre une entreprise qui serait le «Faire innovant» et un consommateur qui serait le «Etre innovant»

\section{Ce n'est pas parce qu'il y a une métaphore qu'on comprend tout le sens !}

"Cela rte fait plus de doute : la sémiologie est opérationnelle et, scientifique ou pas, ça marche!", comme le clame C. Bitoun. De même, F. Bobrie, de s'exclamer : «nous pourrions multiplier les exemples, ils montreraient comment on peut orienter le changement des valeurs, et donc la transformation du récit packaging à partir de n'importe quel point de départ dans le champ isotopique, et comment on peut programmer l'intensité du changement souhaité en fonction des récits préexistants qui structurent ce champ ". La sémiologie, ainsi circonscrite à un enjeu et une démarche bien spécifiques, apparaît de fait comme une méthode infaillible de vérification $\mathrm{du}$ (bon?) sens, et collabore par ce fait à la bonne marche du commanditaire. Nombre d'universitaires présents à ce colloque sont intervenus sur ce point, avertissant d'une éventuelle dérive moralisante d'une part (les discours seraient pré-formatés, les objectifs pas toujours avoués ou clairement formulés, et donc la position du sémiologue un peu partiale), du rêve de la maîtrise du sens absolu d'autre part. J.-D. Urbain (Université de Saint Quentin) interpelle l'assemblée : toutes les fois où l'on a l'occasion de réaliser une métaphore, doit-on entrer en liesse?! Ce n'est pas parce qu'il y a une métaphore que l'on comprend tout le sens !

Ainsi, des limites apparaissent quant à l'identification et l'encodage de sens d'une stratégie d'innovation au sein d'une entreprise.

Si l'innovation se base sur quelque chose de préexistant, le réfèrent est parfois brouillé ; on serait alors dans le neuf absolu et non pas dans l'innovation. Par exemple, Vivendi a opéré une véritable métamorphose en brouillant les référents antérieurs et en relookant les produits ; dans ce cas, l'innovation peut se lire comme un état (la transformation ellemême).

Pour F. Maréchal (Sorgem), "rien ne se crée, tout se transforme", car il y a toujours une analogie avec d'autres univers de sens. Travaillant souvent sur des objets, il affirme qu'à ce niveau, la lecture peut très facilement être brouillée, étant donné la polysémie très fluide de ce type de support : un objet ne relevant pas aussi nettement, de la part de son producteur, d'une intention de communiquer. 
31 Tout changement ou toute nouveauté n'est donc pas innovation : quand un traitement éradiquant le virus du Sida sera trouvé, pourra-t-on dire qu'il s'agit d'une innovation, étant donné l'attente que cette découverte a demandé ?

En outre, si l'innovation n'est signifiante que vis à vis d'un usager, encore faut-il que cet usager participe à ce processus. Pour le cas de l'Euro, s'il réside une quelconque innovation à l'introduction de cette nouvelle monnaie, l'usager n'a pas vraiment le choix comme il a pu, par exemple, davantage l'avoir lors de l'innovation propre à l'introduction du T.G.V. ; il s'agirait, selon N. Couégnas et M.P.Halary (Université de Limoges), "d'une permanence dans le changement. Ce changement de monnaie induit à la fois une conservation et un changement ».

33 Cela dit, la sémiologie gagne du terrain : alors qu'elle était il y a quelques années le plus souvent cantonnée aux phases d'analyse et d'évaluation "a posteriori", ce colloque a démontré l'innovation de la sémiologie, en tant qu'outil d'analyse à hauteur de la stratégie toute entière, depuis la conception stratégique jusqu'aux instruments de communication et leurs applications.

Une grande partie de l'activité du chercheur consiste en la recherche de documentation dans son domaine de compétence et en la communication des résultats de ses travaux, l'écriture ne pouvant s'envisager indépendamment de la lecture. Qu'il s'agisse des réflexions menées, des méthodes employées, des observations effectuées ou des résultats obtenus, la connaissance des travaux scientifiques antérieurs ou en cours constitue une obligation pour le chercheur, afin de délimiter son domaine de recherche par rapport au domaine déjà exploré. Elle représente par ailleurs un stimulant pour sa réflexion et un adjuvant précieux pour ses propres travaux.

Mais l'abondance actuelle des sources d'information ou des matériaux à dépouiller constitue une difficulté croissante que tous les chercheurs des sciences humaines et sociales doivent surmonter. D'autant plus que le transfert des informations peut s'effectuer par différents moyens (relations personnelles entre les chercheurs, livres, revues, congrès, littérature grise, recherche en bibliothèques et en centres de documentation...) qui ne sont pas de valeur équivalente, loin s'en faut.

Dans ce contexte où les supports écrits demeurent toutefois les plus valorisés par les chercheurs, les revues scientifiques incarnent la légitimité scientifique en répondant notamment aux conditions de validation de la production scientifique par la procédure d'évaluation effectuée par des référées généralement anonymes. Elles constituent en outre la source d'actualisation des connaissances et le moyen d'information sur les recherches en cours mené sur un sujet donné. Parmi les nombreux supports existants, elles occupent donc une place centrale dans les pratiques d'appropriation de la recherche, même si les titres ne bénéficiant pas de la même autorité scientifique ne sont pas d'égale notoriété.

Si le chercheur confirmé est censé savoir apprécier la structuration du champ scientifique (P. Bourdieu, 1975) dont il fait partie et valoriser chaque titre de revue en fonction de sa notoriété, de son projet éditorial et des thèmes développés en son sein, qu'en est-il du doctorant, que nous baptiserons jeune chercheur, c'est-à-dire le chercheur terminant son cursus universitaire par le dépôt du travail de longue haleine que constitue la thèse de doctorat dont la production sera d'une importance capitale pour la suite de sa carrière? Celle-ci constitue un moment fort du positionnement du chercheur dans un courant de pensée et d'inscription dans un réseau scientifique. A travers elle, le doctorant doit 
notamment faire la preuve qu'il a su s'approprier les connaissances construites par ses maîtres.

Ce questionnement nous a conduites à nous demander s'il y a eu une évolution des Sciences de l'information et de la communication durant les dernières années fortement marquées par le développement des technologies de l'information et de la communication?

Si nombreuses sont désormais les études qui permettent de mieux caractériser les comportements de lecture des étudiants et notamment leurs relations aux bibliothèques, il n'en est pas de même sur les pratiques des chercheurs en matière de revues scientifiques. Or ces dernières constituent un support de communication qui ne peut être confondu ni avec le livre ni avec la presse d'information, souvent seuls considérés dans ces études. Dans ce cadre, l'étude des publics des revues est une entreprise d'autant plus délicate que les travaux, tant théoriques qu'empiriques, auxquels se référer sont rares, les recherches portant sur les pratiques de lecture des « apprentis chercheurs » concernant les revues scientifiques étant, quant à elles, quasiment inexistantes. Ces usages sont très peu connus y compris par les enseignants chercheurs en Sciences de l'information et de la communication, pourtant grands utilisateurs des revues scientifiques et chargés d'initier les doctorants à la pratique de la recherche.

En conséquence, notre objectif était de déterminer s'il y a valorisation ou non de la revue scientifique par les jeunes chercheurs dans les bibliographies de thèse, cette dernière devant constituer un apport significatif à la discipline scientifique concernée, tant sur le plan théorique que par les résultats publiés.

Quels comportements adoptent donc ces doctorants en matière de recherche documentaire et quelles sources d'information privilégient-ils? Y a-t-il eu une évolution au cours des dix dernières années?

\section{Des pratiques de recherche entre braconnage et bricolage}

La recherche scientifique, tout comme les travaux de bibliothéconomie, ont porté sur les usages d'information des chercheurs confirmés ou des étudiants de premier et de deuxième cycles. La revue est par ailleurs abordée en tant qu'indicateur de l'activité de recherche scientifique et technique.

La sociologie et les sciences de l'éducation se sont penchées sur le comportement et sur les usages de la lecture des étudiants mais en se limitant au support livre.

Notre objectif étant d'appréhender les différentes facettes des démarches suivies par les doctorants, il nous a paru nécessaire d'utiliser un mode d'investigation ouvert et semidirectif, favorisant l'expression spontanée de réflexions personnelles des intéressés sur leurs propres pratiques. Nous avons pour cela mené tout d'abord des entretiens auprès de dix doctorants, inscrits en première, deuxième ou troisième année de thèse à l'Université de sciences humaines de Toulouse Le Mirail, que nous avons ensuite complétés par le questionnement de soixante-neuf doctorants inscrits dans la même université, quelle que soit leur discipline. 


\section{Une représentation pragmatique de la revue scientifique}

45 La représentation que se fait le doctorant de la revue paraît saine. Elle est reconnue comme vecteur privilégié des résultats de la recherche, comme le médium dans lequel les chercheurs publient et est souvent perçue comme organe d'un laboratoire de recherche.

$81 \%$ des étudiants savent ainsi qu'une revue est un périodique où publient des chercheurs et 76,81 \% que des résultats de recherche y sont publiés... même si les uns et les autres ne savent pas donner une définition précise de la revue scientifique en réponse à la question correspondante.

\section{Rencontre avec Robert Escarpit}

Le 23 octobrel998, L'Institut des Sciences de l'Information et de la Communication organisait une journée d'hommage à son créateur, Robert Escarpit. À la fin de cette journée, celui qui fut l'un des fondateurs de la discipline des Sciences de l'Information et de la Communication, prenait la parole.

Hugues Hotier: Robert Escarpit, vous avez patiemment écouté ces hommages, ces éloges et ces louanges, et vous avez été capable, comme on vous l'avait demandé, de ne pas parler, de ne pas protester, de ne pas contredire; mais maintenant vous avez le champ libre et vous pouvez nous dire que nous sommes des ânes et que nous n'avons rien compris, ni à vous, ni à votre œuvre. Si vous disiez cela, il faudrait que l'on recommence tout le mois prochain.

Robert Escarpit: Je ne le dirai pas, je ne le pense d'ailleurs pas. Tout d'abord, j'ai beaucoup de remerciements à faire. Merci à mon ami Hugues Hotier d'avoir organisé ce colloque, merci aussi à ses collaborateurs, à l'ISIC et à Anne-Marie Cocula, la Présidente. Nous avons beaucoup parlé de ce que j'ai fait, mais je voudrais aussi parler de ce que nous avons fait, parce que je n'ai jamais été seul. Tous les gens qui ont travaillé avec moi, il faut que je leur dise merci. Tout d'abord à deux personnes, elles sont d'ailleurs dans la salle, qui ont commencé ce long travail d'organisation, qui a abouti aux recherches que j'ai menées, lorsqu'un ministre, ayant sans doute une petite réserve d'argent, m’a donné quelques sous et deux collaboratrices, l'une qui était ma secrétaire et qui est devenue ma femme, et l'autre, Nicole Robine, qui est aussi dans cette salle et qui a été ma collaboratrice technique pendant toute la période de balbutiements, d'organisation. Je remercie aussi tous mes collaborateurs, qui ont rendu le travail plus agréable, par leur gentillesse, leur disponibilité, je pense à Henri Marquier, à Alain Boisson, à Pierre Orecchioni, à Jean Boussinest. Je pourrais en citer bien d'autres. Je remercie tous les étudiants qui ont suivi mes cours, qui ont passé des thèses avec moi, et qui finalement ont abouti à ce qu'on appelle les Sciences de l'Information et de la Communication. On s'interrogeait sur leur avenir, mais moi je n'ai pas beaucoup de craintes : la discipline est solidement implantée en France. Si j'ai quelques doutes sur ce qui a été dit ce matin, c'est la rançon du succès. En France nous avons l'habitude de couronner un succès par une organisation, une structure ; et dès le début, lorsque j'ai commencé à lancer ces idées un peu follement, j'ai dit : «j'ai une crainte, qu'il y ait un jour une agrégation en Sciences de l'Information et la Communication, un doctorat en Sciences de L'Information et de la Communication » et c'est arrivé : il y a des docteurs, il y a des agrégés en Sciences de l'Information et la Communication. Je n'y peux rien, c'est la nature humaine, c'est la nature aussi de notre pays. Cela dit, il ne faut pas désespérer. Il est certain que les 
dernières décennies ont vu des progrès fantastiques en matière de communication. Je me souviens de l'arrivée de la télévision à Bordeaux, il y a une cinquantaine d'années (cinquante ans et un mois exactement), je me souviens des premiers balbutiements des ordinateurs; l'audiovisuel, dont je disais qu'il n'existe pas - je maintiens qu'il n'existe pas : c'est un concept un peu vague et un peu magique. L'audiovisuel a fait son entrée fracassante, a modifié notre monde, je ne dis pas que tous ces progrès ne sont pas importants : ils le sont, ils ont profondément facilité notre existence et ils ont modifié nos façons de voir, d'aborder les choses; en bien souvent, en mal aussi car il y a un prix à payer pour tout progrès technique. Voyez l'automobile: quels progrès depuis les premières voitures d'il y a un siècle, mais la pollution, elle est là ; les problèmes d'énergie, les problèmes financiers que comporte l'utilisation de l'automobile. C'est un fait que ces maux se sont joints aux progrès techniques.

Robert Escarpit: en matière de communication, il y a eu de fantastiques progrès techniques comme Internet par exemple. Cependant, ce sont des progrès purement matériels. En réalité, nous manions ces outils merveilleux, perfectionnés, avec des cerveaux qui ne sont pas beaucoup plus développés que celui de l'homme de Cromagnon. Nous sommes toujours tributaires du fait que le cerveau humain ne peut pas débiter plus de quarante-cinq bits d'informations par seconde et il y en a des millions à la télévision et des milliards à Internet. Je suis toujours frappé par les métaphores nautiques qu'on emploie pour l'internet: les internautes. C'est un océan d'informations déchaîné qui déferle autour de nous et nous nageons là-dessus comme nous pouvons. Nous n'avons pas encore le secret de la dimension. Nous vivons dans un monde hyper-dimensionnel où les individus se comptent par milliards et tous ceux qui adhèrent à la théorie de l'information savent que l'information est une affaire des cerveaux humains individuels et que c'est chaque cerveau humain qui fabrique son information. Comment se débrouiller dans ce déluge ? C'est encore une question qui n'est pas résolue et j'invite les spécialistes à l'étudier dans les années à venir. Comment se débrouiller pour qu'il n'y ait pas un étranglement de l'information par elle-même, par sa surabondance ? C'est un problème qui m'inquiète beaucoup. Mais enfin on travaille, on se préoccupe. Le colloque d'aujourd'hui en est une preuve ainsi que notre présence: l'information et la communication restent à l'ordre du jour.

On a beaucoup parlé de ma carrière. C'est vrai que j'ai été un touche à tout, et je ne m'en cache pas. L'important n'est pas dans la quantité des choses que l'on touche mais dans la manière de les toucher. J'ai essayé d'être aussi délicat que possible avec les diverses occupations que j'ai remplies. J'ai été très intéressé par ce qui a été dit sur ce que j'ai écrit. Je reconnais qu'il fallait faire des choix, j'ai écrit tellement de choses, des bêtises comme des choses intéressantes. Lorsqu'on m'a parlé de ce colloque, on m'a dit que l'on analyserait les diverses facettes de ma personnalité. J'ai dit: «Mon dieu, il faudrait un miroir aux alouettes comme il y en avait quand j'étais petit ». Quand on le faisait tourner, il y avait des étincelles de tous les côtés, c'était très joli mais on n'y comprenait pas grand chose. Et ce que j'ai finalement produit... ça a une multitude de facettes... Je l'ai écrit quelque part dans un de mes livres, quand on s'exprime, on ne s'exprime pas devant une entité abstraite comme un public. Non, en réalité, on parle à des gens dont ce n'est pas le visage. La situation de communication où nous sommes en ce moment est une situation interactive. Je vois vos visages... je peux lire l'intérêt ou l'ennui sur vos visages. Et je peux 
réagir en fonction de cela. Raconter des histoires, c'est raconter des histoires à quelqu'un, à un public; et c'est pour cela qu'il y a un mot essentiel qui n'a pas été prononcé, sauf dans la toute dernière partie de ce colloque, c'est le mot d'oralité. Quand j'ai commencé à écrire pour les enfants, je n'ai pas écrit pour les enfants, j'ai écrit à mes enfants, les miens, qui avaient un visage, une personnalité, que je connaissais. Et je leur ai raconté des histoires. Bien sûr, ces histoires je les ai fabriquées avec un procédé, en reprenant des expressions du langage pour les Contes de la Saint Glin-Glin, c'est toute l'astuce, de prendre des expressions bizarres de la langue et puis de leur donner une fausse étymologie, et de construire par l'imagination une histoire autour. Bon, j'ai fait ça oralement; et puis un beau jour j'en ai eu assez de raconter sans cesse les mêmes histoires, je les ai tapées à la machine, et je leur ai dit « vous savez lire, vous les lirez ». Et puis ils les ont lues, et ils les ont racontées à leurs enfants, et ce sont mes petits-enfants qui sont venus me trouver pour me demander de terminer. Alors j'ai terminé, et c'est une de mes petites-filles qui m'a suggéré de publier le résultat. Cela a été les Contes de la Saint Glin-Glin. Par la suite, j'ai continué dans la même voie. Pas toujours de manière très heureuse, certaines de mes histoires sont tombées dans un heureux oubli, d'autres, on s'en souvient. J'ai eu le plaisir il y a quelques semaines de recevoir une lettre d'Espagne, m'annonçant que l'on allait traduire en espagnol le Petit Dieu Ocram; Il est né d'une conversation avec mon fils, alors qu'il était un peu plus jeune, il devait avoir six ans. Il m’a dit « et bien voilà, Dieu il s'appelle Ocram», je lui ai dit "il s'appelle Ocram», "oui, oui». Alors je lui ai fait raconter, et après j'ai écrit cette histoire là, et ça a donné un livre qui va être traduit en espagnol, dix ans ou onze ans après. Donc, l'oralité est essentielle. On écrit ce que l'on dit. Et pour compléter ce qui a été dit sur les billets du Monde, cette petite colonne que j'ai remplie...

Pour en revenir à ce billet du Monde, la vérité, c'est que je le disais aux lecteurs, le mécanisme était très simple: j'écoutais la radio, et il y avait une chose, un rapprochement, qui me paraissait cocasse. Alors, ça, c'est une question de tempérament. On a le sens du cocasse ou on ne l'a pas. On trouve des choses comiques ou pas comiques... Ce n'est pas forcément comique, car tous mes billets ne sont pas comiques, ne sont pas faits pour rire. Il y en a qui sont, au contraire, extrêmement tristes. Mais tous ont cette particularité, c'est qu'ils s'adressent au lecteur individuel et lui suggèrent de lire son journal avec cette vision. Une vision naïve, peut-être parce que j'étais un provincial, et qu'un provincial est naïf, c'est ce que disent les Parisiens... Mais j'étais un peu dans la position, vous savez de... je ne sais pas si vous vous souvenez cette histoire du petit garçon qui insulte le roi, lequel s'était dépouillé de ses vêtements, était nu, mais avait exigé que personne ne s'en aperçoive, et il s'est écrié « mais le roi est nu ! », et ça a fait un grand scandale. Et bien, j'étais dans la position de ce petit garçon: je regardais les événements avec naïveté, une naïveté assumée, je ne parle pas du tout de chiqué, je disais «le roi est nu », c'est comme ça, je le disais. C'est ce qui a donné les billets du Monde, jusqu'au moment où cette naïveté m'a abandonné avec l'âge et où je me suis habitué à faire des billets un peu mécaniquement, je rapprochais deux choses, et tac !, il y avait un billet qui sortait de la machine, comme lorsque l'on met une tune dans le bastringue. A ce moment là, ça ne m'a plus amusé, et j'ai demandé à être relevé, c'était en 74, et puis on n'a pas trouvé la relève tout de suite, ce qui fait que j'ai tenu jusqu'en 81 , mes deux derniers billets ont été consacrés à l'élection de François Mitterrand. Voilà en gros ce que je voulais dire là-dessus. 
Hugues Hotier : à propos de ces fameux billets, nous nous demandions s'il y a encore de la place pour l'humour à la une des journaux.

Quand on se pose la question de savoir si l'humour est encore possible dans les journaux, ça dépend. Vous savez, ma théorie de l'information repose sur une idée très simple, c'est que l'information est chez l'auditeur ; la communication est dans la réponse.

Il n'y a pas de communication établie quand vous avez dit « allô », tant qu'il n'y a pas de voix qui réponde « allô ». C'est dans la réponse. Et l'information, c'est ce que vous savez déjà, mais sur lequel vous avez des doutes, ou des incertitudes, et on vous accorde une incertitude provisoire. C'est cela l'information. Cela suppose chez le lecteur une certaine culture, et cette culture est celle que j'appelle de présence au monde, être présent au monde, être présent à ce qui se passe dans le monde. C'est ce que j'ai trouvé de fascinant dans le billet, c'est qu'il fallait que tous les jours je sois présent à ce qui se passait dans le monde, que j'ai conscience de ce qui se passait dans le monde. C'est un réflexe d'ailleurs qui m'est resté ; je suis capable de vous dire ce qui se passe en ce moment au Chili, en Thailande, parce que j'ai prêté attention... j'ai accumulé ça dans ma mémoire. Mais, il y a une histoire qui illustre bien cela, c'est lorsque les soviétiques ont lancé un satellite sur la lune, non habité, et ont déployé un drapeau en métal, un drapeau soviétique, j'ai fait un billet dans le Monde en disant qu'à force de planter des drapeaux sur toutes les planètes, il faudra bien un jour payer les soucoupes volantes; et, un moment après, j'ai reçu un coup de téléphone de Fauvet, qui était alors directeur du Monde, et qui m'a dit « qu'est-ce que c'est cette histoire de planter des drapeaux et de payer les soucoupes ", alors je lui ai dit « vous ne savez pas ce que ça veut dire? », il m'a dit « non, je ne connais pas »; il faut dire que Fauvet est un grand honnête homme, et je lui ai dit « ouvrez la porte, demandez à l'huissier qui est devant la porte s'il sait ce que c'est que payer des soucoupes et planter des drapeaux dans tous les bistrots », et il est revenu et m'a dit «ça y est, j'ai compris maintenant ». C'est simplement parce que j'avais fait une astuce qui ne correspondait pas au monde de Fauvet, il n'était pas au courant.

De toute façon, je suis classé souvent humoriste. Dans le Quid, qui a des réponses à toutes les questions posées dans les concours télévisés, vous trouverez mon nom, et après mon nom, il y a "hum.", humoriste. Dans d'autres publications, je suis classé sociologue, enfin, peu importe. Je veux bien accepter d'être humoriste, mais les vrais humoristes finissent mal. Ils finissent mélancoliques, ils finissent fous, certains même se suicident. C'est le cas du plus grand humoriste américain, Thurdom. Il vivait il y a une cinquantaine d'années, et il a été victime de la vague Mac Carthy aux Etats-Unis, et il s'est suicidé ; il avait 48 ans. Il a publié un livre qui s'appelle My 48 years with Jame Thurdom (Mes 48 ans avec James Thurdom) où il donne ses souvenirs, puis il s'est suicidé. Dedans, il dit « il y a un moment où on ne peut plus être humoriste dans un pays qui fait un adjectif de son identité, on dit que je suis anamerican ", (non américain) alors l'américanisme, est-ce que ça existe? Est-ce qu'on a le droit de parler d'américanisme? Il est heureux de penser qu'on n'a jamais inventé le mot francisme. Enfin, il y a quelqu'un qui a peut-être inventé ce mot là ; mais enfin, il ne connaissait pas non plus Monsieur Le Pen. C'était ce qu'il voulait dire. C'est-à-dire la conformité de ce qu'on appelle le politiquement correct, une pensée unique, une uniformité d'identité avec exclusion de tous les autres, et cela est mortel pour l'humoriste au quotidien; James Thurdom en a tiré de sinistres conséquences.

59 Voilà ce que je voulais dire sur les quelques idées qui me sont passées par l'esprit au cours de ce colloque, qui a été passionnant, passionnant pour ceux qui s'intéressent à ce que j'ai 
fait, et j'espère aussi pour ceux qui ne s'y intéressent pas. C'est à dire que j'ai fait ce que j'ai pu dans ma vie, je me suis bien amusé, je le dis honnêtement, je ne me suis jamais ennuyé et je ne m'ennuie toujours pas. Je disais à un journaliste l'autre jour que participer à ce colloque c'était un peu pour moi comme assister à mes obsèques avec les éloges... Je traduis tout; ce n'est pas du tout des obsèques, c'est au contraire une incitation à vivre, à continuer, et à ne pas changer, si possible. Je vous remercie.

\section{NOTES}

1. Association Internationale pour la Valorisation Interdisciplinaire de la recherche en sciences de l'homme et de la société auprès des Entreprises, Maison des Sciences de l'Homme, Paris 